\title{
SPECTRUM OF COLLECTIVE TRANSVERSE EXCITATIONS IN LIQUID CESIUM NEAR THE MELTING POINT
}

\author{
T. Bryk, I. Mryglod \\ Institute for Condensed Matter Physics, National Academy of Sciences of Ukraine, \\ 1 Svientsitskii Str., Lviv, UA-290011, Ukraine \\ E-mail:mryglod@icmp.lviv.ua \\ (Received December 15, 1997; received in final form April 24, 1998)
}

\begin{abstract}
The spectrum of generalized transverse excitations of liquid cesium at $308 \mathrm{~K}$ is calculated within the approach of generalized collective modes. The transverse "current-current" time correlation function is obtained in the form which satisfies the first frequency moments up to the sixth order. The crossover from the usual hydrodynamic behavior to the regime, where well-defined shear waves are supported by the liquid, is studied. We found the wavenumber $k_{\mathrm{H}}=0.092 \AA^{-1}$ at which the shear propagating modes disappear and transform into two relaxing modes, where one of them is the usual hydrodynamic one. The generalized static shear viscosity is calculated in a wide range of wave numbers $k$. In hydrodynamic limit we found good agreement of the calculated generalized shear viscosity with experimental data.
\end{abstract}

Key words: generalized hydrodynamics, collective modes, shear viscosity, shear waves.

PACS number(s): 61.20.Ja, 61.25.Mv, 61.20.Lc

\section{INTRODUCTION}

The dynamical properties of disordered systems is one of the important problems of modern condensed matter physics. The theoretical investigations of low-frequency excitation spectra are of great importance for understanding the behavior of dynamical structure factors and transport coefficients of disordered media [1]. Especially interesting is the investigation of transverse excitation spectra for amorphous systems and liquids near the melting point. In the last case above the melting point there exists a very small hydrodynamic region of wave vectors and frequency, where the time correlation functions must behave according to the predictions of linear hydrodynamics. However, since the hydrodynamic region is extremely small near the melting point it is quite impossible to reach such small wavenumbers during molecular dynamics (MD) simulations.

Liquid cesium near the melting point has been the subject of numerous theoretical [2-6] and experimental $[7,8]$ studies. The results for spectra of collective excitations and dynamical structure factors [2] showed in general a good agreement with experimental data. The values of calculated transport coefficients for liquid cesium [4] were also found to be in reasonable agreement with experimental ones. The goal of this paper is to investigate in more detail the spectrum of transverse modes and to calculate the transverse "current-current" time correlation function as well as generalized shear viscosity, concentrating the main attention on the range of small and intermediate wavenumbers $k$, where the transition from the usual hydrodynamic behavior to the generalized one takes place. One of the main features of such a transition is the appearance of shear waves, when the value of $k$ becomes larger than some fixed quantity $k_{\mathrm{H}}$. The spectrum of transverse excitations was studied in [2] using a fit- ting procedure to the transverse "current-current" time correlation functions calculated by molecular dynamics. However, these results did not contain the region of small wavenumbers $k$, where the transition to the usual hydrodynamics could be observed. We recall that according to linear hydrodynamics only one diffusive mode exists in the limit of small frequencies $\omega$ and small wavenumbers $k$. This essential drawback of the previous study was due to two main reasons: (i) the value of $k_{\mathrm{H}}$ for the considered thermodynamic point of liquid cesium is too small, so that it is necessary to consider a rather large MDbox in order to be sure that the smallest MD value of $k$ is in the hydrodynamic region; (ii) the fitting procedure used for the calculation of the memory function was constructed in such a form that only the first two nonzero frequency moments for the transverse "current-current" time correlation function had been taken into account.

In our study we follow the generalized collective modes approach which makes possible without any fitting parameters: (i) to take into account more precisely the short-time behavior of time correlation functions and (ii) to perform the calculations for arbitrary $k$-values, even in the hydrodynamic region, interpolating the $k-$ dependence of elements of the generalized hydrodynamic matrix $[9,10]$. The idea of the generalized collective modes approach was suggested initially in [11] and developed later in [12,13]. Mryglod et al. [10,14] formulated this approach in the parameter-free form, and now it is a modern method, which allows one to obtain a selfconsistent description of the dynamical properties of liquids and gases over a wide range of wave-vectors $\mathbf{k}$ and frequencies $\omega$. Within this approach the time correlation functions are presented as a weighted sum of partial terms, each being associated with the relevant collective excitation expressed via eigenvectors and the eigenvalue of a generalized hydrodynamic matrix. For transverse 
fluctuations it has been shown $[14,15]$, that there exists the generalized hydrodynamic mode, the eigenvalue of which tends in the hydrodynamic limit to the result known from standard hydrodynamics. All the other collective modes have finite damping coefficients in the hydrodynamic region and are called the kinetic ones. The number of generalized collective modes in a $M$-mode approximation is equal to the number of independent dynamical variables considered. The time evolution of the dynamic variables may be studied in a computer experiment, and these variables form the basis for the evaluation of relevant time correlation functions. For the first time the three- $[10]$ and four-variable $[14,15]$ approximations have been used for the investigation of the transverse fluctuations of a Lennard-Jones fluid. The general properties of transverse time correlation functions within the $M$-mode approximation of the generalized modes approach has been considered in [15].

For the description of a dense metallic system such a parameter-free generalized collective modes approach has been applied for the first time in [6]. Reasonable agreement with experimental data has been found for the adiabatic speed of sound and the maximum position of the Fourier-transformed longitudinal "current-current" time correlation functions. There were also calculated the generalized thermodynamic quantities - specific heat at constant volume, linear expansion coefficient and ratio of specific heats at constant pressure and at constant volume-as functions of $k$ and it was shown that in the limit $k \rightarrow 0$ these quantities tend to the experimental values. In particular, it was shown, that for a dense metallic system, where the "density-density" time correlation function displays strong oscillations in the region up to $k \sim 1 \AA^{-1}$, the three-variables approximation (hydrodynamic variables only) did not allow the proper description of these oscillations. Within the five-variables approximation there were obtained results which correlated very well with the MD data.

In [16] the parameter-free approach was also applied for the investigation of the "fast sound" phenomenon in a binary $\mathrm{He}_{0.65}-\mathrm{Ne}_{0.35}$ mixture and reasonable agreement with experimental data was obtained. It was shown that the "fast sound" modes, dynamically connected by the light component in the mixture, could be found only for the extended set of dynamic variables which includes in addition to the hydrodynamic variables their first order time derivatives.

In this paper we present an investigation of transverse "current-current" time correlation functions and the generalized shear viscosity for a dense metallic system near the melting point in a wide region of wavevectors including the hydrodynamic region. To extract the transverse excitation spectrum the generalized Langevin equation in the Markovian approximation is solved for transverse "current-current" time correlation function within the precision up to the sixth frequency moment. To our knowledge this is a much better precision in the frequency moments then has ever been obtained in calculations of transverse excitation spectra of liquid metals.

This paper is organized as follows: in section II a short description of the method is given; section III contains the information on the MD simulations and section IV is devoted to the discussion of our results.

\section{THEORETICAL FRAMEWORK}

Let us consider a spatially homogeneous, isotropic system of $N$ identical classical particles of mass $m$ in volume $V$. We start from the definition of the square matrix of time correlation functions $\mathbf{F}(k, t)$. Each element of this matrix is the correlation function of two basis dynamic variables:

$$
F_{i j}(k, t)=\left\langle A_{i}(\mathbf{k}, 0) A_{j}^{*}(\mathbf{k}, t)\right\rangle .
$$

Here $M$ microscopic dynamic variables $A_{i}(\mathbf{k}, t)$ form the basis set $\left\{A_{i}(\mathbf{k}, t)\right\}$ for the definition of $M \times M$ square matrix $\mathbf{F}(k, t)$.

The matrix equation for the Laplace transform $\tilde{\mathbf{F}}(k, z)$ of $\mathbf{F}(k, t)$ can be written in a well-known form (see, e.g. [17])

$$
[z \mathbf{I}-i \Omega(k)+\tilde{\mathbf{M}}(k, z)] \tilde{\mathbf{F}}(k, z)=\mathbf{F}(k, 0),
$$

where $i \Omega(k)$ and $\tilde{\mathbf{M}}(k, z)$ are the frequency matrix and the matrix of the memory functions, respectively. In the Markovian approximation for the memory functions, when $\tilde{\mathbf{M}}(k, z) \simeq \tilde{\mathbf{M}}(k, 0)$, one has

$$
[z \mathbf{I}+\mathbf{T}(k)] \tilde{\mathbf{F}}^{(m)}(k, z)=\mathbf{F}(k, t=0),
$$

where

$$
\mathbf{T}(k)=-i \Omega(k)+\tilde{\mathbf{M}}(k, 0)=\mathbf{F}(k, 0) \tilde{\mathbf{F}}^{-1}(k, 0)
$$

is the so-called generalized hydrodynamic matrix, $\tilde{\mathbf{F}}^{(m)}$ denotes the Laplace-transformed matrix of time correlation functions to be found in the Markovian approximation, and I is the identity matrix. Using (3) it is straightforward to derive the following equalities

$$
\begin{aligned}
& \int_{0}^{\infty} \mathbf{F}^{(m)}(k, t) d t=\int_{0}^{\infty} \mathbf{F}(k, t) d t, \\
& \mathbf{F}^{(m)}(k, t=0)=\mathbf{F}(k, t=0),
\end{aligned}
$$

which are important from the point of view of sum rules and connect the moments of time correlation functions $\mathbf{F}^{(m)}(k, t)$ and $\mathbf{F}(k, t)$.

The matrix equation (3) can be solved analytically in terms of the eigenvalues $z_{\alpha}(k)$ and the eigenvectors $\hat{X}_{\alpha}=\left\{X_{i, \alpha}\right\}$ of the matrix $\mathbf{T}(k)(4)$, 


$$
\sum_{j=1}^{M} T_{i j}(k) X_{j, \alpha}=z_{\alpha}(k) X_{i, \alpha}, i=1, \ldots, M
$$

so that we obtain

$$
\tilde{F}_{i j}^{(m)}(k, z)=\sum_{\alpha=1}^{M} \frac{G_{i j}^{\alpha}(k)}{z+z_{\alpha}(k)}
$$

where

$$
G_{i j}^{\alpha}(k)=\sum_{l=1}^{M} X_{i \alpha} X_{\alpha l}^{-1} F_{l j}(k, 0)
$$

is the weight coefficient describing the contribution of the mode $z_{\alpha}$ in the function $\tilde{F}_{i j}^{(m)}(k, z)$. In the timerepresentation one has the form

$$
F_{i j}^{(m)}(k, t)=\sum_{\alpha=1}^{M} G_{i j}^{\alpha}(k) \exp \left\{-z_{\alpha}(k) t\right\}
$$

Hence, as is seen from (10), the time correlation function $F_{i j}^{(m)}(k, t)$ within the generalized mode approach can be expressed as a weighted sum of $M$ exponential terms, and each term of this sum is connected with the relevant effective collective mode $z^{\alpha}(k)$. The precision of the Markovian approximation in $M$-modes description is controlled by the equalities (5) and (6). Let us discuss the last statement in more details for the case of transverse fluctuations considered here.

The basis set of dynamic variables for the investigation of transverse fluctuations $\mathbf{A}(k, t)$ consists of the transverse current operator $J(k, t)$,

$$
J(k, t) \equiv J_{t}(k, t)=\frac{1}{\sqrt{N}} \sum_{i=1}^{N} m v_{i}^{t} \exp \left(i \mathbf{k r}_{i}(t)\right),
$$

and its first time derivatives up to the $s$-th order, namely,

$$
\begin{aligned}
& \mathbf{A}(k, t)=\left\{A_{i}(\mathbf{k}, t)\right\} \\
& =\left\{J(k, t), \dot{J}(k, t), \ddot{J}(k, t), \ldots,\left(i L_{N}\right)^{s} J(k, t)\right\},
\end{aligned}
$$

where $\dot{J}(k, t)=i L_{N} J(k, t), \ddot{J}(k, t)=\left(i L_{N}\right)^{2} J(k, t)$ and $i L_{N}$ is the Liouville operator. Thus, we have

$$
A_{i}(\mathbf{k}, t) \equiv\left(i L_{N}\right)^{i-1} J(k, t), \quad i=1,2, \ldots, s+1
$$

The notations $\mathbf{r}_{i}, v_{i}^{t}$ in (11) denote a position and a transverse component of velocity of the $i$ th particle, respectively.

In the hydrodynamic limit we can restrict ourselves to considering the operator of transverse current alone. In this case the well-known single-exponential form for $F_{J J}^{(m)}(k, t)$ with the real hydrodynamic eigenvalue $z_{h} \simeq$ $D k^{2}$ can be reproduced from (10). In the intermediate range of wavenumbers $k$ and frequencies $\omega$, where the short-time kinetic properties become more important, one has to consider an extended set of dynamic variables. We note that as it was proved previously [15] the first $2 s+1$ frequency moments (including the zeroth one) coincide with the explicit values for the function $F_{J J}^{(m)}(k, t)$ when we use set (12). Moreover, as follows from (5), $\tilde{F}_{J J}^{(m)}(k, 0)=\tilde{F}_{J J}(k, 0)$. In our study we shall consider the basis set of dynamic variables (12) with $s=3$ which consists of the current operator and its first three time derivatives. This means that the first frequency moments of the function $F_{J J}^{(m)}(k, t)$ are explicitly reproduced up to the sixth order. This result can be improved in a systematic way by taking into account higher-order time derivatives in (12).

Taking $m=1$ the $4 \times 4$ real symmetric matrix of static correlation functions $\mathbf{F}(k)=\mathbf{F}(k, t=0)$ for $s=3$ has the form

$$
\mathbf{F}(k)=\left(\begin{array}{cccc}
F_{J J}(k) & 0 & -F_{j . j}(k) & 0 \\
0 & F_{j . j}(k) & 0 & -F_{j . j}(k) \\
-F_{j . j}(k) & 0 & F_{\tilde{J} . j}(k) & 0 \\
0 & -F_{\ddot{J} . j}(k) & 0 & F_{J . j}(k)
\end{array}\right)
$$

The matrix $\tilde{\mathbf{F}}(k)=\tilde{\mathbf{F}}(k, z=0)$ is antisymmetric, namely,

$$
\tilde{\mathbf{F}}(k)=\left(\begin{array}{cccc}
\tau_{J J}(k) F_{J J}(k) & F_{J J}(k) & 0 & -F_{j . j}(k) \\
-F_{J J}(k) & 0 & F_{j J j}(k) & 0 \\
0 & -F_{j . j}(k) & 0 & F_{J j}(k) \\
F_{j . j}(k) & 0 & -F_{J j}(k) & 0
\end{array}\right)
$$


where

$$
\tau_{J J}(k)=\frac{1}{F_{J J}(k, 0)} \int_{0}^{\infty} F_{J J}(k, t) d t,
$$

is the transverse correlation time $[10,14]$.

It should be emphasized that the approach, described above, does not contain any fitting or adjustable parameters. Just the Markovian approximation is made in order to obtain equation (3). The applications of this method for a Lennard-Jones fluid showed $[10,14,15]$ very reasonable results for dynamical properties.

\section{MOLECULAR DYNAMICS SIMULATIONS}

We have performed MD simulation for liquid cesium with mass density $\rho=1832.1 \mathrm{~kg} / \mathrm{m}^{3}$ at temperature 308 $\mathrm{K}$ and at constant volume $V=L^{3}$ using a system of 500 particles. The effective two-body oscillating potential $\Phi_{i j}(r)$ was obtained from the pseudopotential theory using a simple Ashcroft empty-core pseudopotential [19] with the parameter $r_{c}=2.72$ a.u. For the local-field correction the parameterization of Ichimaru-Utsumi [20] was taken. The potential was calculated in tabular form on a grid with a mesh size of $0.04 \AA$. In order to take into account the Fridel oscillations, the cut-off radius $r_{c u t}$ was chosen to be $18.12 \AA$, in the vicinity of sixth node. This potential had been used previously [2] for the study of the static and dynamic properties of liquid cesium and good agreement with experimental data was observed. The reduced interatomic potential and the pair distribution function $g(r)$ are plotted in the fig. 1 .

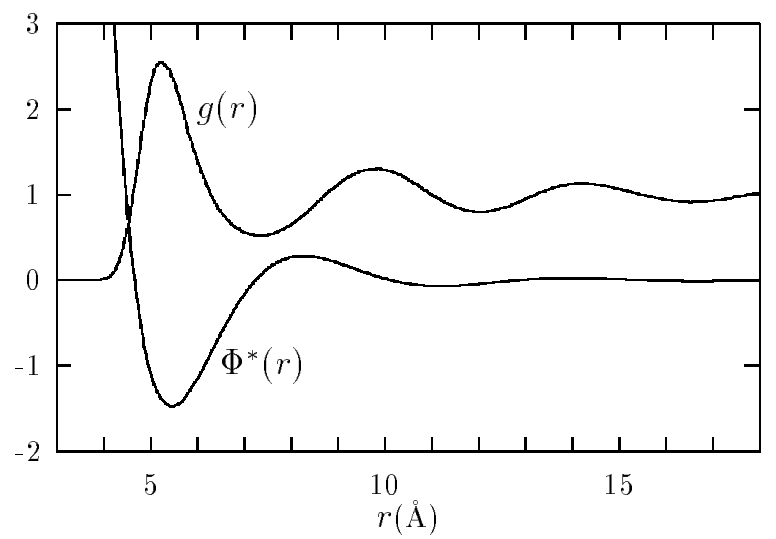

Fig. 1. The pair distribution function $g(r)$ and the reduced potential $\Phi^{*}=\Phi_{i j} / k_{B} T$.

The equations of motion were integrated by means of a fourth-order predictor-corrector Gear algorithm with the time increment of $\Delta t=8 \times 10^{-15} \mathrm{~s}$. The initial configuration of particles was the face-centered cubic lattice and the initial velocities were randomly put to Maxwell distribution. The melting of the initial configuration and the following thermalizing were performed in 19500 time steps. The observation time over the equilibrium state of the system was 2400 ps (300000 time steps). The energy conservation for the MD run was less than $0.02 \%$, due to the value of $\Delta t$, the grid size of the tabulated potential and the sophisticated integration method. Every sixth configuration was taken into account by computation of static equilibrium averages. The time correlation functions were calculated by shifting time origins $\left(\Delta t_{0}=6 \Delta t\right)$ on the grid of 1024 points with the step of $3 \Delta t$. Additional averaging of correlation functions was performed over all the $N_{k}$ possible vectors $\mathbf{k}$ (with $|\mathbf{k}|=k$ ), which are compatible with the reciprocal lattice of the MD-box due to periodic boundary condition. The smallest possible $k$-value $k_{\text {min }}=2 \pi / L$ reached during the MD run was $0.16029 \AA^{-1}$.

\section{RESULTS AND DISCUSSION}

The method of generalized collective modes allows to perform the investigation of dynamical properties of liquids operating with the static correlation functions only. One exception is the correlation time (15) which appears in the matrix $\tilde{\mathbf{F}}(k, z=0$ ) (see (14)). For the calculation of $\tau_{J J}(k)$ we used the definition $(15)$ where the function $F_{J J}(k, t)$ was obtained directly by the MD experiment.

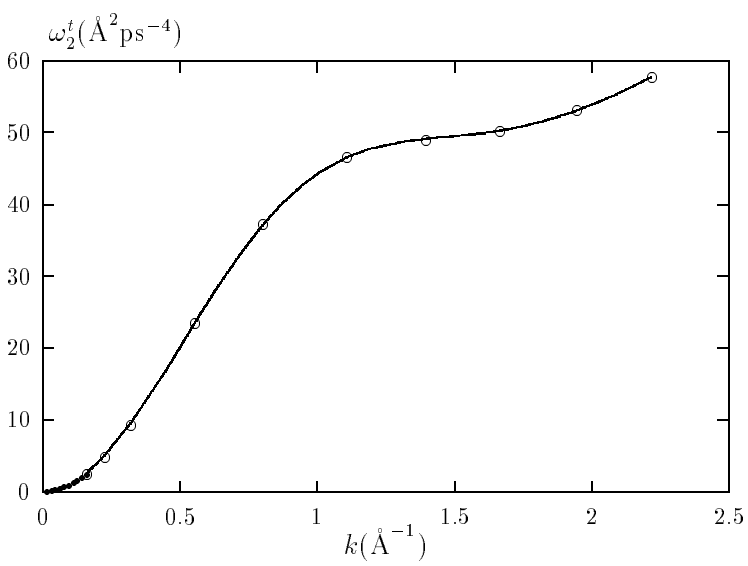

Fig. 2. The second moment $\omega_{2}^{t}(k)$ of the transverse "current-current" time correlation function. Values calculated directly in MD and via pair distribution function are shown by open circles and full line, respectively. Extrapolated values for $k \rightarrow 0$ are shown by closed circles.

The diagonal elements $F_{A_{i} A_{i}}(k)$ of matrix (13) are connected with the frequency moments $\omega_{2(i-1)}^{t}$ of transverse "current-current" time correlation function $F_{J J}(k, t)$ [18]. All the matrix elements (except $F_{\ldots j}(k)$ ) have been calculated directly in MD. The static correlation functions $F_{J . J}(k)$ has been found as numerical second time 
derivative of the function $F_{\ddot{J} .}(k, t)$ at $t=0$. In fig. 2 the MD second moment $\omega_{2}^{t}(k)$ (circles) is shown in comparison with the data obtained from the definition $\omega_{2}^{t}$ via the pair distribution function (solid line). It is seen that the agreement is rather good. The fourth frequency moment $\omega_{4}^{t}(k)$ of the transverse "current-current" time correlation function $F_{J J}(k, t)$ can be also calculated by using the definition via three-particle distribution function [18]. However, this demands in fact extremely large computational efforts. We have calculated directly in MD the static correlation function $F_{\ddot{J} . J}(k)$ which is simply connected with $\omega_{4}^{t}(k)$. In figure 3 the $k$-dependent function $F_{\ddot{J} \ddot{J}}(k) / k^{2}$ is shown by open circles.

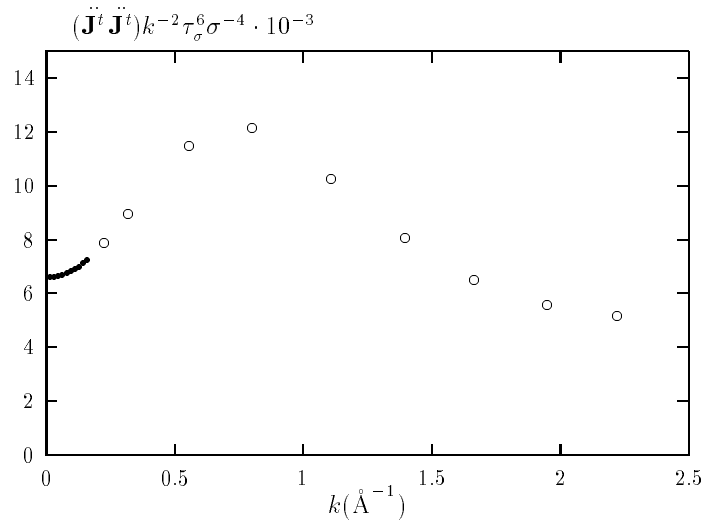

Fig. 3. The reduced static correlation function $F_{j}(k) / k^{2}$ calculated by MD (open circles). The extrapolated values for $k \rightarrow 0$ are shown by closed circles. The length and time scale units are $\tau_{\sigma}=4.494 \mathrm{ps}$ and $\sigma=k_{\text {min }}^{-1}=6.239 \AA$, respectively.

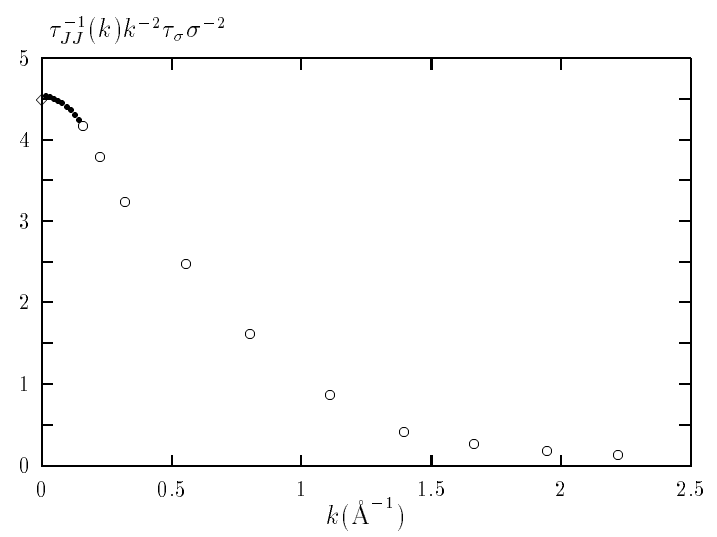

Fig. 4. The reduced inverse correlation time $\tau_{J J}^{-1}(k)$ divided by $k^{2}$ (open circles). The extrapolated values for $k \rightarrow 0$ are shown by closed circles. Diamond at $k=0$ is the calculated value (17) using experimental data for shear viscosity [21]. The length and time scale units are the same as for fig. 3.
In order to study the dynamical properties in the range of small wavenumbers $k$ we extrapolated the functions $\omega_{2}^{t}(k)$ and $\omega_{4}^{t}(k)$ using the MD data as well as symmetry properties of static correlation functions. The extrapolated results for $\omega_{2}^{t}(k)$ and $\omega_{4}^{t}(k)$ when $0<k<k_{\text {min }}$ are shown by closed circles in fig. 2 and fig. 3 , respectively.
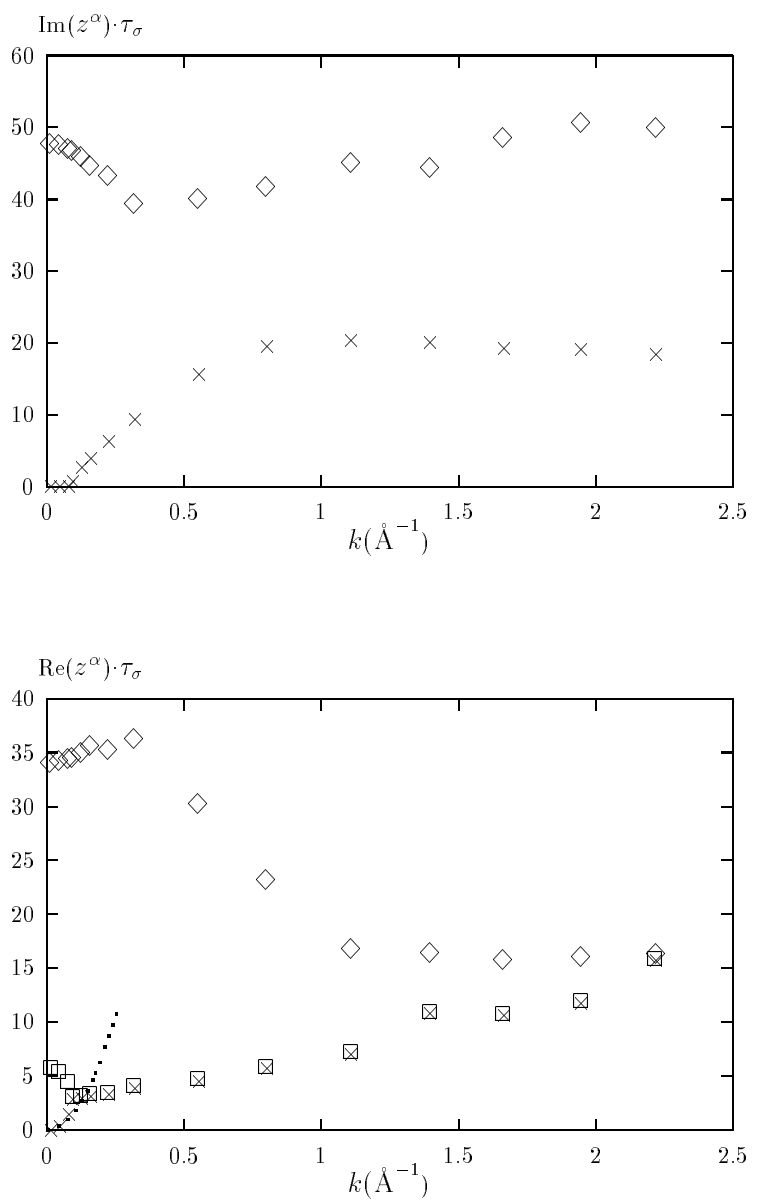

Fig. 5. The spectrum of generalized collective modes: imaginary (a) and real (b) parts of reduced eigenvalues. Dotted curve displays the hydrodynamic behavior (19) with the experimental value of shear viscosity $\eta$ [21]. The time scale unit is the same as for fig. 3 .

It is well-known that in the hydrodynamic limit the transverse "current-current" function $F_{J J}(k, t)$ of a simple liquid has the following form [18]:

$$
F_{J J}(k, t)=A \exp \left(-t / \tau_{J J}^{h}\right)
$$

where the hydrodynamic correlation time is

$$
\tau_{J J}^{h}=\frac{\rho}{\eta k^{2}}
$$



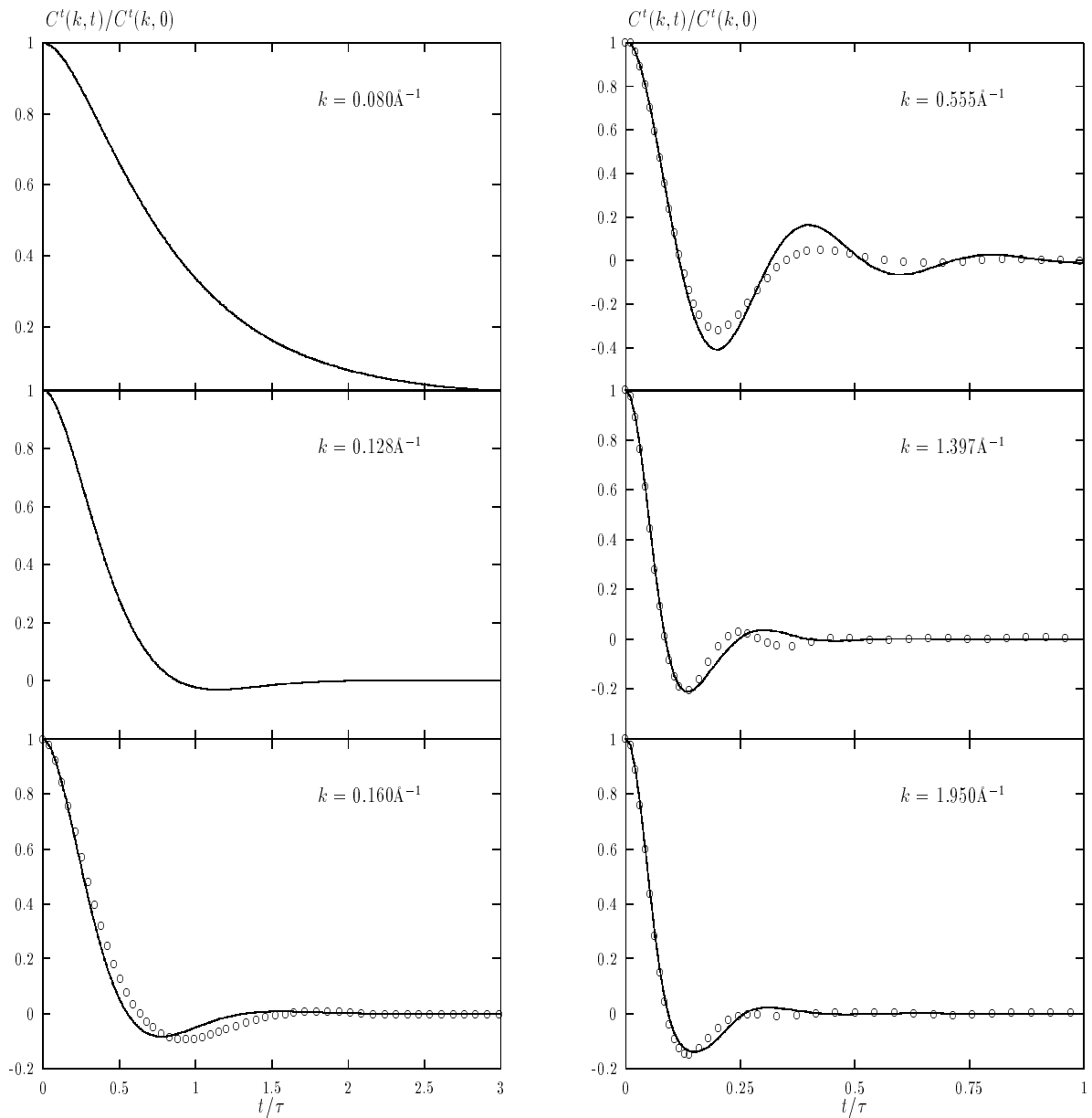

Fig. 6. Normalized transverse "current-current" time correlation function $C(k, t)$ for the six fixed wavenumbers $k$. Solid lines: generalized mode approach, open circles: MD data.

$\rho$ and $\eta$ are the mass density and shear viscosity, respectively. In order to study the range of small wavenumbers we have extrapolated the MD data found for $\tau_{J J}^{-1} k^{-2}$ in assumption that this function behaves as $c_{1}+c_{0} k^{2}$. The constants $c_{1}$ and $c_{0}$ have been estimated from two smallest MD $k$-points. The results for $\tau_{J J}^{-1} k^{-2}$ are shown in fig. 4. Closed circles denote the extrapolated data for $0<k<k_{\text {min }}$, and the MD data are plotted by open circles. It is seen in fig. 4 that the point at $k=0$ (diamond) obtained from (17) with the experimental value of shear viscosity $\eta[21]$ correlates very well with our data.

The results for the generalized transverse collective modes obtained in the four-variable approximation are shown in fig. 5. For $k>0.092 \AA^{-1}$ the spectrum of transverse excitations consists of two pairs of complexconjugated propagating modes with

$$
z_{j}^{ \pm}(k)=\sigma_{j}(k) \pm i \omega_{j}(k), \quad j=1,2 .
$$

The high-frequency modes have comparable real parts (dampings) of eigenvalues with low-frequency ones only for $k>1 \AA^{-1}$ (fig. 5, b). For smaller $k$-values the contribution from $z_{2}^{ \pm}(k)$ into all dynamical processes is very small and in the hydrodynamic limit it becomes negligibly small. When $k$ becomes smaller $k=0.092 \AA^{-1}$ the lower-lying propagating mode disappears and transforms into two relaxing modes with purely real eigenvalues (see fig. 5, a and 5, b). In the limit $k \rightarrow 0$ the eigenvalue of one of these modes tends to a finite damping coefficient, while the second eigenvalue in full agreement with the hydrodynamic description (see (16)) behaves as

$$
\left.z^{h}(k)\right|_{k \rightarrow 0}=\frac{1}{\tau_{J J}^{h}}=\frac{\eta}{\rho} k^{2} .
$$

In fig. 5, b the dotted line displays the dependence (19) with the experimental value of $\eta$. It is seen that this curve almost coincides with crosses below the point where the transverse propagating modes disappear.

It should be stressed that in contrast with the previous theoretical works on liquid cesium we were able within the approach of generalized collective modes to find the $k$-point $k_{H}=0.092 \AA^{-1}$ at which transverse propagating modes transform into the relaxing ones: the relaxing kinetic and diffusive hydrodynamic modes (19) (boxes and crosses in fig. 5, b, respectively). 

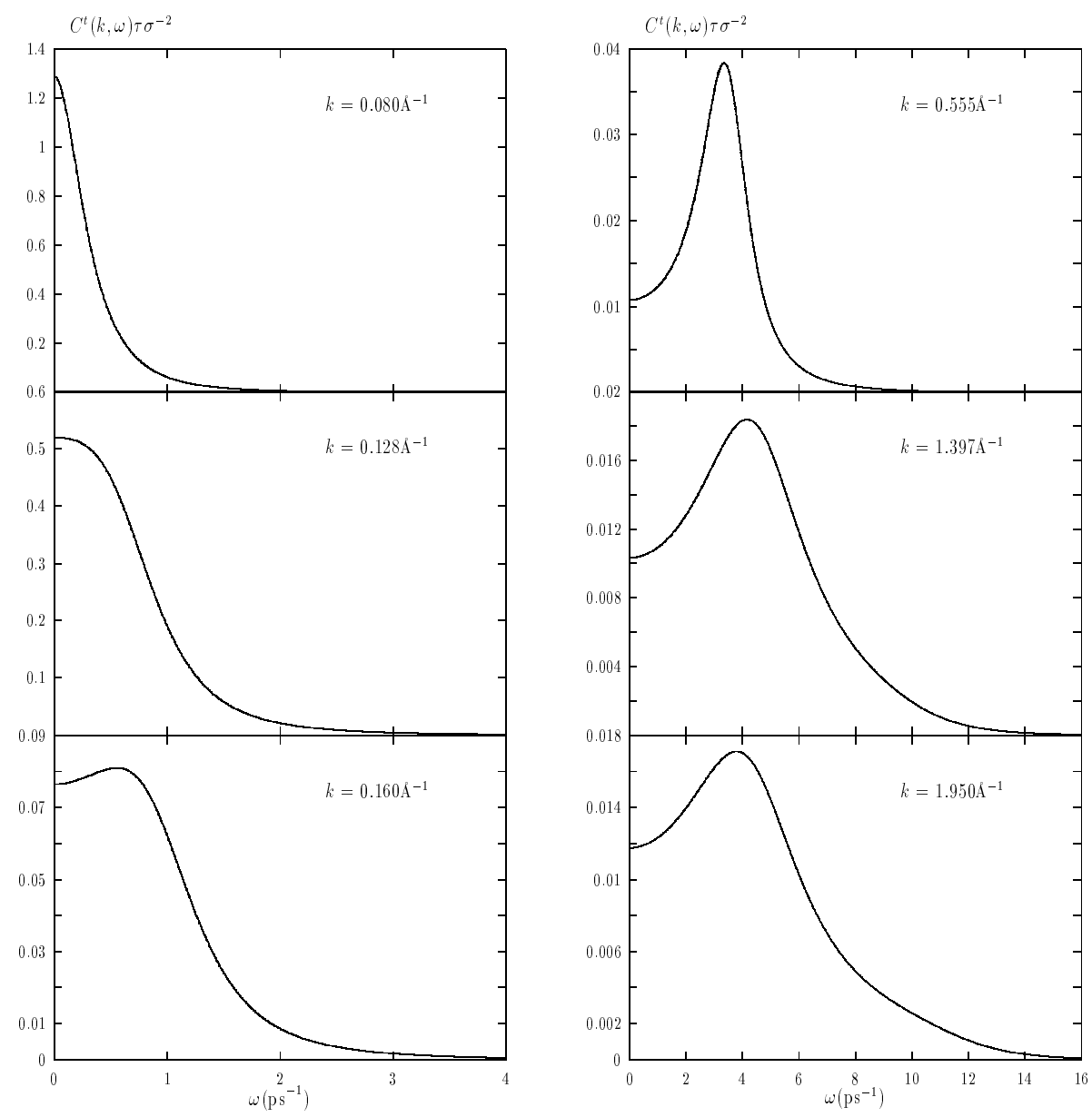

Fig. 7. The Fourier-spectra of transverse "current-current" time correlation function $C^{t}(k, \omega)$ for six fixed values of $k$. The length and time scale units are the same as for fig. 3 .

The behavior of the transverse "current-current" time correlation function for several fixed values of $k$ is shown in fig. 6. The MD data are given by open circles and the results, calculated within the generalized modes approach, are plotted by solid curves. For the value $k=$ $0.080 \AA^{-1}$ (which is much smaller than the MD-value $k_{m i n}$ and is also less than $k_{H}$ ) the normalized function

$$
C(k, t)=F_{J J}(k, t) / F_{J J}(k, 0)
$$

is nearly a simple exponential function, as it should be in hydrodynamic region [18]. When wavenumber $k$ becomes larger than $k_{H}$, the behavior of $C(k, t)$ changes essentially: one can observe (see figure $6 \mathrm{~b}$ ) the appearance of a small time range (near $t \sim 1 \mathrm{ps}$ ) where $C(k, t)$ is negative. This means that the system is already beyond the hydrodynamic region and the appearance of propagating modes causes negative values of $C(k, t)$. The oscillations of transverse "current-current" time correlation functions become more pronounced for larger values of $k$ (see figures $6 \mathrm{c}$ and $6 \mathrm{~d}$ ). When the wavenumber $k$ further increases the propagating modes become over-damped causing the decrease of the amplitude of the first minimum.
In the fig. 7 we display the behavior of Fourier-spectra of transverse "current-current" time correlation functions. We would like to remind that within the approach of generalized collective modes we have $M$ (in the present study $M=4)$ contributions to $C^{t}(k, \omega)$ with weight coefficients $(9)$. For $k<k_{H}$ the functions $C^{t}(k, \omega)$ are single Lorentzian (see (8)) centered at origin, because highfrequency kinetic propagating modes do not make any visible contribution in the whole $k$-region investigated. For $k>k_{H}$ the functions $C^{t}(k, \omega)$ have a peak centered at $\omega>0$. The strong contribution of pronounced noncentral Lorentzian is the consequence of propagating modes with small damping (connected with a real part of relevant eigenvalue $z^{\alpha}$ ). Due to increasing of the damping coefficient for $k>1 \AA^{-1}$ the peak-structure of $C^{t}(k, w)$ become less pronounced.

The shear viscosity as the transport coefficient may be generalized as a function of $k$ and $\omega$ using the relation between transport coefficients and memory functions [15]

$$
\begin{aligned}
& \tilde{\eta}(k, z=i \omega)=\eta^{\prime}(k, \omega)-i \eta^{\prime \prime}(k, \omega) \\
& =\frac{\rho}{k^{2}} \tilde{\varphi}_{J J}^{(t)}(k, z=i \omega),
\end{aligned}
$$


where the memory function can be expressed in terms of transverse "current-current" time correlation function $F_{J J}(k, t)$

$$
\tilde{\varphi}_{J J}^{(t)}(k, z=i \omega)=\frac{1}{\int_{0}^{\infty} e^{-i \omega t} F_{J J}(k, t) d t}-i \omega .
$$

The dimensionless static shear viscosity

$$
\eta(k)=\eta^{\prime}(k, \omega=0)=\frac{\rho}{k^{2} \tau_{J J}(k)}
$$

is shown in fig. 8. The experimental value at $k=0$ is given by diamond. We obtained the value of $0.725 \times$ $10^{-3} \mathrm{~Pa} \cdot s$ that is in good agreement with the experimental data of $0.70 \times 10^{-3} \mathrm{~Pa} \cdot s$ [21]. We would like to note that the generalized static shear viscosity $\eta(k)$ within the generalized modes approach could be considered as a parameter of theory. This quantity is directly connected with the correlation time $\tau_{J J}(k)$ which has been calculated in the MD simulations. However, for the calculation of $\tau_{J J}(k)$ the usual phenomenological relaxationtime procedure can be also used (see, for instance, [22]). Applying such a scheme we found that in the intermediate range of wavenumbers, when $0.4 \AA^{-1}<k<1.5 \AA^{-1}$, the value of $\eta(k)$ is distingtly larger thanthat, obtained within phenomenologic treatment. This result is known in the literature the so-called "shoulder" problem [22] and will be discussed in more detail in the subsequent study [23] where a new approximation for the calculation of a memory function will be proposed.

We conclude by the following remarks:

(i) Applying the approach of generalized collective modes without any fitting parameters to dense metallic cesium we have obtained the spectrum of transverse collective excitations and found the $k$-point $k_{\mathrm{H}}$ where the lowest-lying propagating modes disappear transforming into two relaxing modes. For $k>k_{\mathrm{H}}$ these propagating modes describe the transverse shear waves observed in the behavior of "current-current" time correlation function. When $k<k_{\mathrm{H}}$ one of the relaxing modes in the hydrodynamic limit has the eigenvalue proportional to $k^{2}$ with the hydrodynamic coefficient $\eta / \rho$ which is very close to the experimental estimated value, and the second one is the relaxing kinetic mode. (ii) The transverse "current-current" time correlation function, found within the four-variables approximation, satisfies the first sum rules up to the sixth order frequency moment. Moreover, in the used scheme the value $F_{J J}(k, \omega=0)$ of the Fourier transform of this function at $\omega=0$ coincides with the explicit one.

(iii) We have used a simple extrapolation scheme to investigate the transverse excitation spectrum in the region $k<k_{\min }$, that has not been accessible in molecular dynamics. The reliability of extrapolation procedure is shown by calculations of generalized static shear viscosity $\eta(k)$. In the hydrodynamic limit the value of static shear viscosity has been found to be in good agreement with the experiment.

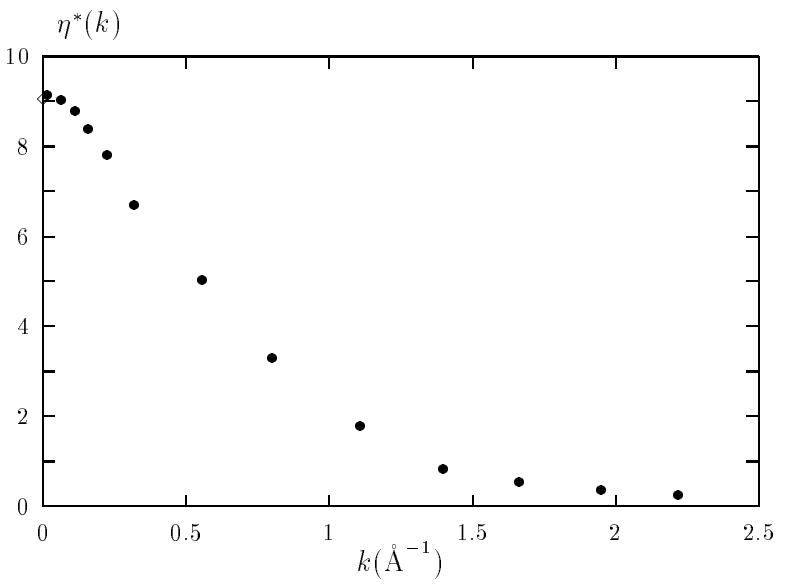

Fig. 8. The generalized static shear viscosity $\eta^{*}(k)$ (in reduced units). Diamond at $k=0$ displays the experimental point [21]. $\eta^{*}(k)=\eta(k) \tau_{\sigma} \sigma m^{-1}$, where $m$ is the atomic mass of cesium and $\tau_{\sigma}, \sigma$ are the same as for fig.3.

\section{ACKNOWLEDGMENTS}

The basic codes for the MD simulations have been taken from the SIMUL package, which is the product of the Institute for Theoretical Physics, Technische Universität Wien. One of us (I.M.) acknowledges support by the Österreichische Fonds zur Förderung der wissenschaftlichen Forschung under Proj. No. P12422-TPH.
[1] Dynamics of disordered materials, edited by Richter D., Petry W., Dianoux A. J., Texteira J. (Springer: Berlin, Heidelberg, 1989).

[2] S. Kambayashi, G. Kahl, Phys. Rev. A 463255 (1992).

[3] S. Kambayashi, G. Kahl, Europhys. Lett. 18, 421 (1992).

[4] U. Balucani, A. Torcini, R. Vallauri, Phys. Rev. A 46 , 2159 (1992)

[5] U. Balucani, A. Torcini, R. Vallauri, Phys. Rev. B 47.
3011 (1993).

[6] T. Bryk, Y. Chushak, J. Phys.: Cond. Matt. 9, 3329 (1997).

[7] T. Bodensteiner, C. Morkel, W. Gläser, B. Dorner, Phys. Rev. A 45, 5709 (1992); T. Bodensteiner, Ph.D. Thesis, TU München (1990).

[8] Ch. Morkel, T. Bodensteiner, H. Gemperlein, Phys. Rev. E 47, 2575 (1993). 
[9] P. Westerhuijs, W. Montfrooij, L. A. de Graaf, I. M. de Schepper, Phys. Rev. A 45, 3749 (1992).

[10] I. M. Mryglod, I. P. Omelyan, M. V. Tokarchuk, Mol. Phys. 84, 235 (1995).

[11] W. E. Alley, B. J. Alder, Phys. Rev. A 27, 3158 (1987).

[12] B. Kamgar-Parsi, E. G. D. Cohen, I. M. de Schepper, Phys. Rev. A 35, 4781 (1987)

[13] I. M. de Schepper, E. G. D. Cohen, C. Bruin, J. C. van Rijs, W. Montfrooij, L. A. de Graaf, Phys. Rev. A 38, 271 (1988).

[14] I. P. Omelyan, I. M. Mryglod, Cond. Matt. Phys. (Lviv) iss. 4, 128 (1994); I. M. Mryglod, I. P. Omelyan, Phys. Lett. A 205, 401 (1995).

[15] I. M. Mryglod, I. P. Omelyan, Mol. Phys. 90, 91 (1997).

[16] T. Bryk, I. Mryglod, G. Kahl, Phys. Rev. E 56, 2903
(1997).

[17] J.-P. Hansen, I. R. McDonald, Theory of Simple Liquids (Academic, London, 1986).

[18] J. Boon, S. Yip, Molecular Hydrodynamics (McGrawHill, New-York, 1980).

[19] N. W. Ashcroft, Phys. Lett. 23, 48 (1966).

[20] S. Ichimaru, K. Utsumi, Phys. Rev. B 24, 7381 (1981).

[21] R. Ohse, Handbook of Thermodynamic and Transport Properties of Alkali Metals (Blackwell Scientific, Oxford, 1985).

[22] U. Balucani, R. Vallauri, T. Gaskell, Phys. Rev. A 35, 4263 (1987).

[23] I. Mryglod, A. Hachkevych, Ukr. Fiz. Zh., 1998 (in press).

\title{
СПЕКТР УЗАГАЛЬНЕНИХ ПОПЕРЕЧНИХ ЗБУДЖЕНЬ У РІДКОМУ ЦЕЗІї ПОБЛ ИЗУ ТОЧКИ ПЛ АВЛЕННЯ
}

\author{
Т. Брик, І. Мриглод \\ Інститут фізики конденсованих систем НАН Украйни, \\ вул. Свениіцького, 1, Львів, 290011, Украйна \\ E-mail:mryglod@icmp.lviv.ua,me.: 761157, фaкc:761978
}

\begin{abstract}
У підході узагальнених колективних мод виконано розрахунок спектра узагальнених поперечних збуджень рідкого цезію при температурі 308 K. Знайдено вираз для поперечної часової кореляційної функції "потік-потік" у формі, для якої викону ються правила сум до шостого порядку. Вивчено перехід від звичайної гідродинамічної поведінки до ре жиму, у якому спостерігаються добре визначені зсувні хвилі. Знайдено значення хвильового вектора $k_{\mathrm{H}}=0.092 \AA^{-1}$, при якому зсувні пропагаторні хвилі пропадають $\mathbf{i}$ трансформуються у дві релаксаційні моди, одна з яких є відомою гідродинамічною модою. Виконано розрахунок узагальненої статичної зсувної в'язкости в широкій області зміни модуля хвильового вектора $k$. У гідродинамічній гранищі знайдено добре узгодження з даними експерименту .
\end{abstract}

